\title{
Les spécificités de la biologie et de son enseignement
}

VIIes rencontres européennes de didactique de la biologie

Daniel Favre

\section{OpenEdition}

\section{Journals}

Édition électronique

URL : http://journals.openedition.org/trema/1962

DOI : 10.4000/trema.1962

ISSN : 2107-0997

Éditeur

Faculté d'Éducation de l'université de Montpellier

Édition imprimée

Date de publication : 1 septembre 1996

Pagination : 1-2

ISSN : 1167-315X

Référence électronique

Daniel Favre, "Les spécificités de la biologie et de son enseignement », Tréma [En ligne], 9-10 | 1996,

mis en ligne le 11 septembre 2013, consulté le 22 septembre 2020. URL : http://

journals.openedition.org/trema/1962 ; DOI : https://doi.org/10.4000/trema.1962

Ce document a été généré automatiquement le 22 septembre 2020.

Trema 


\title{
Les spécificités de la biologie et de son enseignement
}

\author{
VIIes rencontres européennes de didactique de la biologie
}

Daniel Favre

1 Le thème principal mais non exclusif retenu pour ces Rencontres a été: "Les spécificités de la Biologie et de son enseignement». Ce thème nous a permis de commencer à mieux préciser en quoi l'enseignement de la biologie diffère de celui des autres disciplines scientifiques et de mieux identifier les compétences que cet enseignement permet (ou pourrait permettre) de développer chez les élèves et chez les étudiants. L'organisation de ces journées a tenté d'optimiser les conditions favorisant la communication et le débat scientifique. Le nombre des conférences a été réduit et celles-ci réservées à quelques intervenants extérieurs à notre Association, connus pour la qualité de leurs travaux et susceptibles d'introduire le thème de cette année. Ainsi, John Stewart a abordé les problèmes épistémologiques associant la biologie et la cognition, et Jean Claude Hervé nous a exposé les différentes logiques qui sont à l'œuvre dans la conception des programmes scolaires en Biologie.

2 Nos Rencontres ont privilégié les tables rondes sur le thème central, et des ateliers " implicatifs ». Une séance de présentation de posters portait sur d'autres aspects de la recherche actuelle en Didactique de la Biologie, tandis que des temps de discussions ont été réservés à des recherches en cours posant problème. Les apports des cinquante-cinq participants furent riches, complémentaires, et ont souvent traduit leur passion vis à vis de la Biologie et des enjeux multiples de son enseignement.

3 Les discussions confrontèrent les modèles de scientificité utilisés actuellement dans l'enseignement et les programmes de la Biologie avec ce que la majorité des participants a semblé considérer comme une spécificité de cet enseignement: le développement de "l'esprit naturaliste». Cet état d'esprit réunit nombre de sous disciplines de la Biologie et se traduit notamment par la capacité à effectuer des approches systémiques : qu'il s'agisse d'écosystèmes ou d'homéostasie, de dynamique de populations ou d'interactions cellulaires, de duplication ou d'autoréparation du génome. 
Les nouveaux programmes d'enseignement permettent-ils de faire croître ces capacités chez les élèves ? Comment éviter le morcellement des connaissances ? À quelle période de la scolarité doivent avoir lieu les synthèses ? Comment évaluer leur appropriation? Ne donne-t-on pas trop d'importance aux fonctionnements moléculaires, à la génétique? La biologie, science de la vie et du vivant, communique inévitablement à travers son enseignement une image de la vie et de l'homme. Laquelle?

5 Si cette image ne nous convient pas, pouvons-nous faire évoluer cet enseignement en développant ses spécificités? Cette question ne semble pas se poser avec la même acuité dans tous les pays européens. Les collègues présents ont souvent trouvé les Français très « dépendants et soumis » aux programmes. Ils nous ont ainsi rappelé que les programmes d'enseignement dans plusieurs pays européens n'étaient qu'un ensemble de repères et de conseils mais qu'il appartenait à chaque enseignant de traiter telle ou telle partie du programme et de choisir les angles d'approche et les dispositifs didactiques qui lui semblaient les plus appropriés.

Cette liberté par rapport aux programmes ne serait-elle pas une condition pour que les enseignants puissent, sans malaise (et tout en tenant compte du programme national pour les Terminales), enseigner la Biologie selon leur sensibilité, permettre aux élèves et étudiants de développer des capacités spécifiques à cet enseignement et inviter ainsi la « passion » à être au rendez-vous de la Biologie?

\section{AUTEUR}

\section{DANIEL FAVRE}

Laboratoire de modélisation de la relation pédagogique, équipe ERES, Université de Montpellier II 